\title{
La vivienda vernácula en Burkina Faso: transformaciones de los modos de habitar de las culturas del Sahel
}

\author{
Vernacular Housing in \\ Burkina Faso: Transformations \\ of Sahel Cultures' Ways of Inhabiting
}

\author{
MARÍA AGUILAR SÁNCHEZ \\ Universidad de Sevilla, España \\ JOSÉ MANUEL ALMODÓVAR MELENDO \\ Universidad de Sevilla, España
}

Resumen: Burkina Faso es un país de África subsahariana con un alto índice de pobreza que, al igual que otros países del Sahel, ha triplicado su población en el último medio siglo, al pasar de 4.5 a 18 millones de habitantes. Estos cambios demográficos, unidos a las migraciones y a la importación de nuevos materiales de construcción, han modificado los modos de vida tradicionales. Como resultado, las nuevas tipologías de viviendas que se están implantando masivamente en la periferia de las ciudades difieren en gran medida de la arquitectura tradicional. Tras varias estancias de larga duración y seis meses de trabajo de campo, se analizan diferentes modos de habitar de las culturas mos-

Recepción: 2 de diciembre de 2019. / Aceptación: 27 de abril de 2020. 
si y peul. Los resultados muestran que la introducción de la propiedad de la tierra, la especulación en el sector constructivo y la cada vez mayor escasez de recursos naturales están obligando a estas culturas a modificar sus modos de vida.

Palabras clave: vivienda vernácula; Burkina Faso; Sahel; clima; materiales de construcción.

Abstract: Burkina Faso is a country in sub-Saharan Africa with high poverty rates, which has tripled its population in the last half century like many countries in the Sahel region. More specifically, it has increased from 4.5 to 18 million inhabitants. These demographic changes, combined with an intense migration and the import of new building materials, are modifying traditional ways of life. As a result, new housing typologies that are being built in the city outskirts differ greatly from traditional architecture. After several long-term stays and six months of fieldwork, the authors have explored different ways of life of the Mossi and Peul cultures. Results show that introduction of land ownership, real-estate speculation, and an increasing scarcity of natural resources are forcing these cultures to change their ways of life.

Keywords: vernacular housing; Burkina Faso; Sahel; climate; building materials.

\section{Introducción}

La palabra vivienda simboliza en muchas lenguas africanas el clan o el linaje como concepto de nosotros, así comienza el proceso de identificación y de afiliación o pertenencia. Por tanto, cuando surgen problemas en torno al concepto de vivien$\mathrm{da}$ en una sociedad africana, éstos se trasladan al de la propia identidad (Monnier y Droz 2004, 20-22).

En el caso de las viviendas de Burkina Faso, las técnicas de construcción tradicional constituyen un importante legado al que no se le está prestando la atención adecuada. Prueba de ello son los escasos grupos de repercusión que investigan tales técnicas o el patrimonio arquitectónico en África Occidental, 
a excepción de los grupos CRAterre y la Association La Voûte Nubienne. Perteneciente al primero, Basile Kéré (1995) ha sido de los pocos autores que ha mostrado la diversidad del patrimonio arquitectónico y los diferentes tipos de hábitats de este país. Cabe citar también el trabajo coordinado por JeanBaptiste Kiéthega (2006), que destaca por el estudio detallado de tradiciones y conocimientos ancestrales, y es un importante referente que contribuye a la conservación del patrimonio y a la permanencia de la identidad cultural local.

Aunque la historia precolonial de Burkina Faso no está exenta de conflictos, la colonización dejó marcada la herencia de los pueblos nativos que habitaban el territorio desde hacía siglos y cambió completamente la estructura del territorio (Ouattara y Some 2009, 23-26). Tras la independencia de Francia en 1960, el neocolonialismo actual tampoco está contribuyendo a la protección de la cultura local. Todo esto está generando una transformación en los modos de vida y en las tipologías de viviendas que se construyen, las cuales difieren cada vez más de la herencia secular. A pesar de todo, los peuls y los mossis han sabido mantener sus identidades como pueblos mediante sus expresiones artísticas, religiosas, de lenguaje y gastronómicas. Sin embargo, la supervivencia de estos pueblos y de sus identidades se ve aún más amenazada ahora, ya que a esta convergencia de factores hay que sumarle el conflicto terrorista del Sahel y el cambio climático, los cuales están afectando radicalmente a la población de Burkina Faso (Navarro 2019). Así pues, cabe analizar las causas de esta pérdida de patrimonio arquitectónico y de transformación de los modos de habitar, así como las consecuencias o los efectos que conlleva.

\section{Finalidad y pertinencia}

El presente trabajo se centra en la investigación de viviendas tradicionales y modos de habitar de las dos culturas mayoritarias en las franjas central y norte del país, mossi y peul respec- 
tivamente. El objetivo general es arrojar nueva luz sobre las causas y los efectos de las transformaciones que experimentan, teniendo en cuenta la influencia del clima, los factores antropológico-culturales y el contexto socioeconómico actual. Como objetivos específicos, se busca aportar información reciente sobre tipologías de viviendas tradicionales de estas dos culturas. Asimismo, buscamos identificar las dificultades que éstas encuentran para mantener sus costumbres y tradiciones relacionadas con sus modos de habitar, y las causas que están propiciando estos cambios, especialmente los que llevan al abandono o la transformación de las construcciones tradicionales. Para ello se plantean las siguientes preguntas: ¿cómo son y cómo se construyen las viviendas vernáculas de los peuls y los mossis?, ¿por qué se están modificando sus modos de habitar en la actualidad?

Debemos tener en cuenta que la arquitectura vernácula del Sahel y de áreas colindantes es un buen ejemplo de cómo la interacción armoniosa entre el clima, los materiales locales, las técnicas de construcción y las tradiciones puede generar soluciones arquitectónicas con valores positivos en términos de calidad ambiental e identidad cultural. Constituyen un conjunto de sistemas con los que una cultura ha interactuado con el medio usando los limitados recursos disponibles en un momento específico o lugar geográfico, para obtener las condiciones adecuadas de habitabilidad y garantizar su supervivencia en situaciones extremas.

La mayoría de los estudios sobre arquitectura vernácula se han centrado en aspectos meramente formales o compositivos. En contadas ocasiones se hace referencia a su relación con el medio ambiente o con valores culturales locales (Almodóvar 2006). Afortunadamente, esta tendencia ha ido cambiando en los últimos años, y, en nuestros días, los conceptos y las técnicas tradicionales están siendo considerados por muchos arquitectos como una fuente de conocimiento e inspiración para diseñar soluciones más eficientes y para conservar los recursos naturales y el entorno ambiental (Almodóvar, Pinheiro y Souza 2011, 17). 
Por todo ello, consideramos pertinente el estudio de las viviendas peul y mossi como una contribución a la permanencia de las identidades culturales y del patrimonio arquitectónico de Burkina Faso que aún sobrevive. Identificar las causas de los cambios en los modos de habitar permite, además, reflexionar sobre las repercusiones en los comportamientos como sociedad. Esta investigación se plantea desde la perspectiva que revalora el estudio de la psicosociología del espacio, donde la generación de los espacios habitacionales y urbanos es de especial importancia para comprender las conductas humanas (Baringo 2013; Lefebvre 1974).

\section{Metodología y enfoque}

Para alcanzar los objetivos planteados se utilizó una metodología cualitativa y multidisciplinar en la que se incluyeron herramientas propias de la arquitectura y la sociología. Por un lado, se emplearon métodos técnicos y gráficos, como la elaboración de planos y la identificación de materiales para el levantamiento de las viviendas; por otro, herramientas como la observación participante y las entrevistas en profundidad, para obtener datos en relación con los modos de habitar y las reflexiones sobre las causas y las consecuencias de las transformaciones en la actualidad. Este procedimiento, en el que se combina la expresión visual y la escrita, fue utilizado por Mauss (1905) para extraer conclusiones generales sobre el comportamiento de las sociedades a partir de pequeñas muestras de población.

Tras varios años de convivencia con la sociedad burkinabé, entre 2013 y 2017, se seleccionó como muestra de población para el presente trabajo una comunidad seminómada asentada en el norte de la región Plateau-Central ${ }^{1}$ de Burkina Faso. Se

${ }^{1}$ La estructura administrativa es como la francesa: una "región” está compuesta por varias provincias que, a su vez, se dividen en municipios. Dado que la nomencla- 
estableció luego un plan de visitas para hacer las entrevistas y tomar datos de las viviendas. Dicha información específica se obtuvo entre octubre y diciembre de 2017. En lo que respecta a las entrevistas, los datos se tomaron directamente en lengua vernácula, en este caso el mooré y el pular, ${ }^{2}$ y las llevaron a cabo sociólogos locales especializados en el contacto y la sensibilización de comunidades rurales y que, además, transcribieron las entrevistas de la lengua local al francés. Durante las visitas a las viviendas, la autora no tuvo dificultades para entenderse con la población local, puesto que ya había convivido con población mossi y peul desde 2013. Los entrevistados mostraron total disposición a contestar las preguntas formuladas y a compartir momentos de su vida cotidiana y de sus tradiciones.

\section{Estructura del artículo}

En consonancia con la metodología planteada, este trabajo se estructura en tres fases. En primer lugar, se analizan factores geográficos y climáticos para entender el entorno natural. Teniendo en cuenta esos aspectos, se estudian tipologías de viviendas tradicionales, en particular su morfología y sus dimensiones, materiales y sistemas constructivos. El trabajo está centrado en lo tipológico y se analiza la estructura de la vivienda tradicional y su conexión con los modos de habitar de la cultura local. Finalmente, se discuten los cambios que se están produciendo, para obtener conclusiones en relación con sus causas y sus efectos.

tura de los niveles de división territorial difiere según el país, utilizaremos el término región para referirnos a la zona que agrupa una o más provincias.

${ }^{2}$ Hay más de sesenta lenguas en Burkina Faso, aunque sólo algunas son oficiales, como la mossi (o mooré), la diula (o yulá) y la fulfulde (o pular). La principal lengua oficial utilizada por las instituciones es el francés, introducido durante la colonización (de 1895 a 1960). 


\section{Entorno natural y factores socioeconómicos que influyen en los modos de habitar}

El Sahel se encuentra situado al sur del desierto del Sahara y constituye una zona de transición tanto geográfica como climática que afecta el tipo de vegetación y los recursos naturales disponibles. Esta franja ocupa parcialmente el norte de Burkina Faso, un país en el que convergen dos tipos principales de clima que condicionan de forma muy diferente el modo de habitar y las tipologías arquitectónicas: cálido y húmedo en la zona sur, y cálido y seco en el área central y norte (mapa 1) (Kottek et al. 2006, 261).

Las viviendas peul y mossi se construyen en el área central y norte del país, en condiciones de clima cálido y seco, en el que se distinguen dos variantes: árido (BWh) ${ }^{3}$ y semiárido (BSh). ${ }^{4}$ Aunque en el área situada más al norte se observa un paisaje desértico, en la mayor parte de esta zona predomina la estepa, conformada por arbustos bajos, árboles y artemisas (Piesik 2017).

Este artículo se centra en el análisis de las tipologías de viviendas de la zona central, ya que es ahí donde conviven las culturas seleccionadas. En concreto, se seleccionaron casos de estudio dentro de la franja intermedia (BSh) (Shmite y Nin 2015).

El cambio climático está teniendo un gran impacto en las culturas que conviven tanto en el Sahel como en áreas limítrofes, ya que son muy sensibles a la disponibilidad de recursos debido a que las condiciones son cada vez más extremas. Las interacciones entre suelo, vegetación y atmósfera alteran considerablemente las precipitaciones y alargan el periodo de sequías y su concentración en tiempos cortos. Esto está provocando la erosión del suelo y, por tanto, afectando

${ }^{3}$ Nomenclatura según la clasificación climática de Köppen, donde las siglas corresponden a $\mathrm{B}=$ clima seco, $\mathrm{W}$ = árido, $\mathrm{y} \mathrm{h}=$ cálido.

${ }^{4}$ Nomenclatura según la clasificación climática de Köppen, donde las siglas corresponden a $\mathrm{B}=$ clima seco, $\mathrm{S}=$ semiárido, $\mathrm{y} \mathrm{h}=$ cálido. 
la biodiversidad y la disposición de recursos naturales. Investigaciones como la de Breil, Panitz y Schädler (2017) demuestran la enorme repercusión de este proceso en África Occidental y las consecuencias que está teniendo el cambio climático en los modos de habitar tradicionales de Burkina Faso, cuyo ciclo de vida está completamente ligado a la naturaleza. En este sentido, las culturas del Sahel han adoptado diferentes tipos de viviendas móviles para mantener su modo de vida no sedentario. Estas construcciones están realizadas con materiales que provienen de la explotación de los recursos forestales y, por tanto, están muy condicionadas por la disponibilidad de estos recursos (Ganaba, Ouadba y Bognounou 2004, 11-17).

Mapa 1. Localización del Sahel y zonas climáticas (2019), Burkina Faso

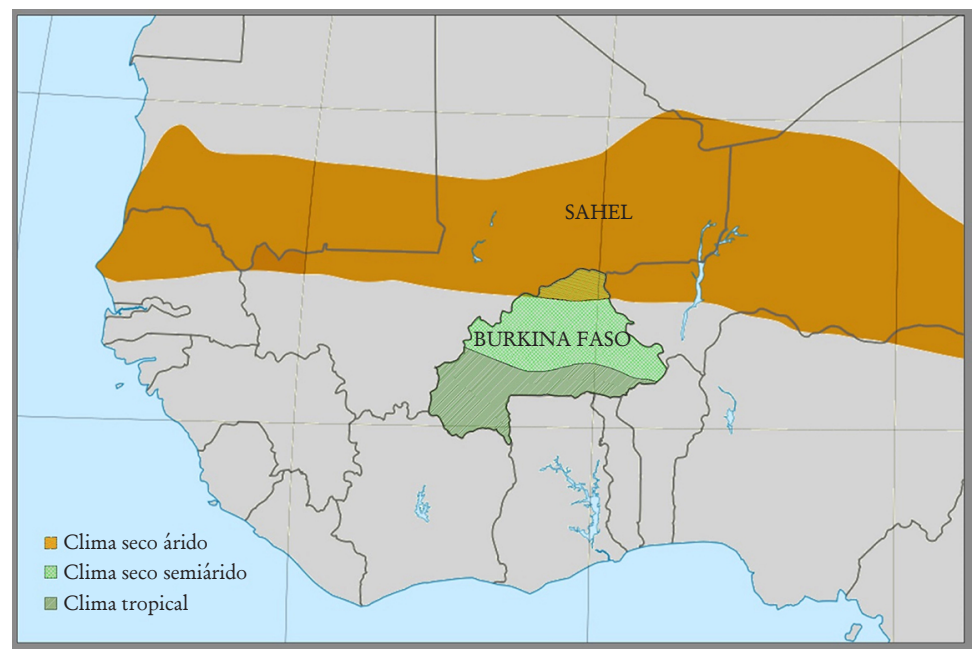

Elaboración propia, 2019. 
Burkina Faso es uno de los países más pobres del mundo ${ }^{5}$ $y$ actualmente se encuentra inmerso en una crisis alimentaria cada vez más grave. Por otro lado, los conflictos terroristas que han tenido lugar en Mali (Mendoza 2013, 22) están afectando las fronteras del país, por lo que la población se ha visto sometida a un aislamiento territorial. En relación con la amenaza terrorista: "Aparte de Mali, Burkina Faso (creo que ahora especialmente), Níger y Chad son países directamente amenazados por la actual situación" (Navarro 2019, 137). A estos graves conflictos debe añadirse la degradación ambiental antes mencionada (Shmite y Nin 2015, 205-219).

Los problemas particulares relacionados con la vivienda se ven acentuados en este contexto tan complejo. El sueño de poseer una vivienda en propiedad se extiende por todo el país, ya que se asocia a tener un mínimo de seguridad y estabilidad, es una garantía frente a los bancos y constituye, además, un signo de éxito social (Wyss 2005).

Aunque el país está sumido en la escasez de recursos y la inestabilidad política, es muy interesante antropológicamente debido a la gran variedad de culturas que conviven en la misma nación (Baux 2013). Consideramos que esta diversidad de etnias de los pueblos burkinabés se traduce, asimismo, en una gran riqueza arquitectónica, ya que todavía conservan en gran medida sus modos de vida y sus tradiciones ancestrales. En este sentido, cabe destacar que el último Censo General de Población y Vivienda de Burkina Faso de 2006, conocido como RGPH, ${ }^{6}$ refleja que $26.3 \%$ de la población del país habita en viviendas tradicionales (Sagnon y Sawadogo 2009, 53). ${ }^{7}$

${ }^{5}$ En 2018, se encontraba en el número 183 de los 187 países clasificados por IDH de las Naciones Unidas, y la esperanza de vida era de 58-59 años. El IDH de 2018 era de 0.423 .

${ }^{6}$ Recensement Général de la Population et de l'Habitation.

7 Clasificadas en el RGPH-2006 como "cases". El informe respalda la transición hacia la construcción de viviendas con "materiales duros", el hormigón y la chapa corrugada. 


\section{Tipologías de viviendas tradicionales en Burkina Faso}

En Burkina Faso hay tres tipos principales de hábitats que han condicionado la configuración de las tipologías de las viviendas vernáculas (Kiéthega 2006). En términos generales, y sin tomar en cuenta algunos casos particulares, se distinguen los siguientes tipos de construcciones:

- Hábitat temporal: construcciones de las etnias seminómadas del norte del país, el Sahel burkinabé.

- Hábitat sedentario moaga: construcciones de planta circular y cubierta cónica de paja, en clima semiárido, principalmente del centro y el este del país.

- Hábitat sedentario tropical: construcciones de planta ortogonal y cubierta de argamasa de la zona sur y suroeste.

En esta investigación nos centramos en dos tipos de construcciones: el hábitat temporal y el hábitat sedentario moaga, ya que se corresponden con un clima cálido y seco. ${ }^{8}$ En ambos predominan ejemplos de viviendas tradicionales de los pueblos mossi y peul, ya que son las etnias mayoritarias en estas regiones, y, por tanto, los hemos seleccionado como casos de estudio.

\section{La vivienda temporal peul}

\section{El pueblo peul del Sabel burkinabé}

Las tres provincias de la región del Sahel de Burkina Faso son Oudalan, Soum y Séno (con capitales en Gorom-gorom,

${ }^{8}$ En el norte de Burkina, zona que corresponde al Sahel, se distinguen claramente un clima y un entorno natural diferentes a los del resto del país (mapa 1). Sin embargo, el no sedentarismo de las etnias que habitan esta zona hace que la diferencia en cuanto a culturas se produzca de manera más gradual. De hecho, a medida que se avanza hacia el norte, hay una presencia mayor de viviendas temporales, ya que los pueblos de origen no sedentario, como los peuls, migran de norte a sur según necesidades. 
Djibo y Dori), y limitan con Mali y Níger. Esta región está habitada por grupos étnicos diferentes, de los cuales los fulanis (o peuls) constituyen la mayor parte; aproximadamente 6.8\% de la población del país (INSD 1994). ${ }^{9}$

Los fulanis son uno de los pueblos mayoritarios conocidos por ser grandes trashumantes (Halen 2012). Como a otros pastores del Sahel, se les asocia particularmente a las migraciones a gran escala, ya que se mueven a distancias considerables con rebaños de ganado en busca de pastos y agua. Sin embargo, desde las importantes sequías de principios de la década de 1970, cuando el número de rebaños se redujo drásticamente, los fulanis del norte de Burkina Faso han disminuido su área de movimiento y han participado cada vez más en la migración temporal a las ciudades. La propensión a migrar no es uniforme en todos los fulanis y se asocia a variables como la edad, la actividad económica, el tamaño y la composición del hogar y el subgrupo étnico (Hampshire y Randall 2000, 247).

La procedencia de este pueblo es difícil de ubicar, puesto que hay peuls en diferentes países, aunque la mayoría de las fuentes sitúa su origen en Mali. Se distinguen por los rasgos físicos, la lengua y el estilo de vida no sedentario basado principalmente en el ganado. Barral (1967) define a los nómadas puros como aquellos que sólo viven del ganado, por lo que en el caso de los peuls burkinabés puede considerarse que practican un seminomadismo. Las principales actividades económicas en el área donde viven son el pastoreo extensivo y la agricultura de secano, con cultivos de mijo y sorgo. No hay sistema de riego, por lo que fuera de la temporada de lluvias es imposible trabajar la tierra, y esto es lo que hace tan importante al ganado. Los fulanis poseen sobre todo ganado vacuno, ovino y caprino.

Los peuls del Sahel burkinabé conviven con otros pueblos con modos de vida seminómada, como los bellas y los tuaregs, e incluso con población sedentaria, principalmente compuesta

\footnotetext{
${ }^{9}$ Instituto Nacional de Estadística y Demografía de Burkina Faso.
} 
por los songhais y los mallebés (Barral 1967, 10). Entre los mismos peuls del norte de Burkina Faso, hay diferentes subgrupos étnicos. Por ejemplo, el origen de los que viven en la provincia de Oudalan no es el mismo que el de los que proceden de Séno (Dori). Del mismo modo, en Oudalan hay peuls provenientes de Gao y otros de Djelgodji. En términos generales, hay cuatro subgrupos étnicos principales de los fulanis en esta región: djelgobes, liptaakos, riimaaibes liptaakos y gaobes (Barral 1967, 14).

\section{Tipología de vivienda y modos de habitar}

Los pueblos que actualmente conservan pautas de vida seminómada son los bellas, los peuls y los tuaregs. Sin embargo, cada vez les resulta más difícil mantener sus hábitos y sus costumbres ancestrales.

Las cabañas de los bellas y los tuaregs difieren en tamaño y forma de las que construyen los peuls, pero, debido a los requerimientos del modo de vida trashumante, todas tienen en común el uso de materiales ligeros y un carácter efímero, lo que se traduce en economía de materiales y rapidez de construcción. Por este motivo, el uso de esteras de paja se ha identificado con la tradición nómada. El cambio paulatino de los modos de vida hacia pautas más sedentarias, que lleva implícito el paso de una vivienda temporal a una definitiva, está influyendo en el tipo de materiales utilizados en la construcción y, por lo tanto, afecta de forma determinante la configuración de las tipologías tradicionales.

De las tres culturas de tradición seminómada del norte del país citadas anteriormente, nos centramos en el análisis de la peul, que es la etnia mayoritaria en el país debido a que sus flujos migratorios llegan más al sur que los que siguen los bellas y los tuaregs. Así, los peuls son los que más interactúan con otras etnias sedentarias de Burkina Faso, tanto en los espacios habitados como en las actividades cotidianas. Los peuls destacan 
IMAGEN 1. Conjunto de viviendas peuls del Sahel burkinabé, Burkina Faso
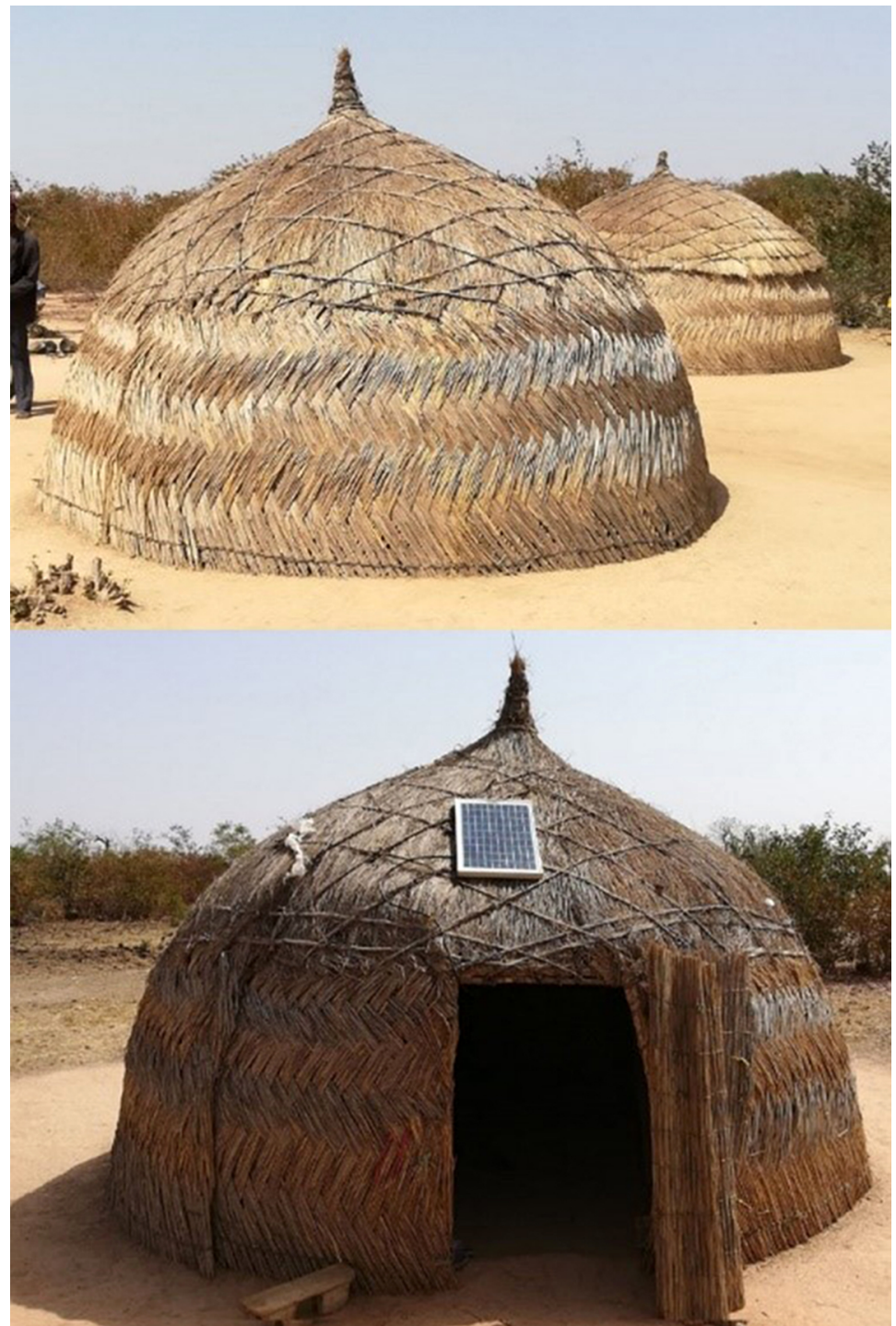

Fotografía de María Aguilar Sánchez, 2017. 
por ser grandes comerciantes de ganado, al que siempre llevan consigo en sus desplazamientos (imagen 2). Estos animales suponen su principal medio de vida y les aportan leche, el alimento básico de su dieta alimenticia.

IMAGEN 2. Viviendas peuls del Sahel burkinabé y entorno, Burkina Faso

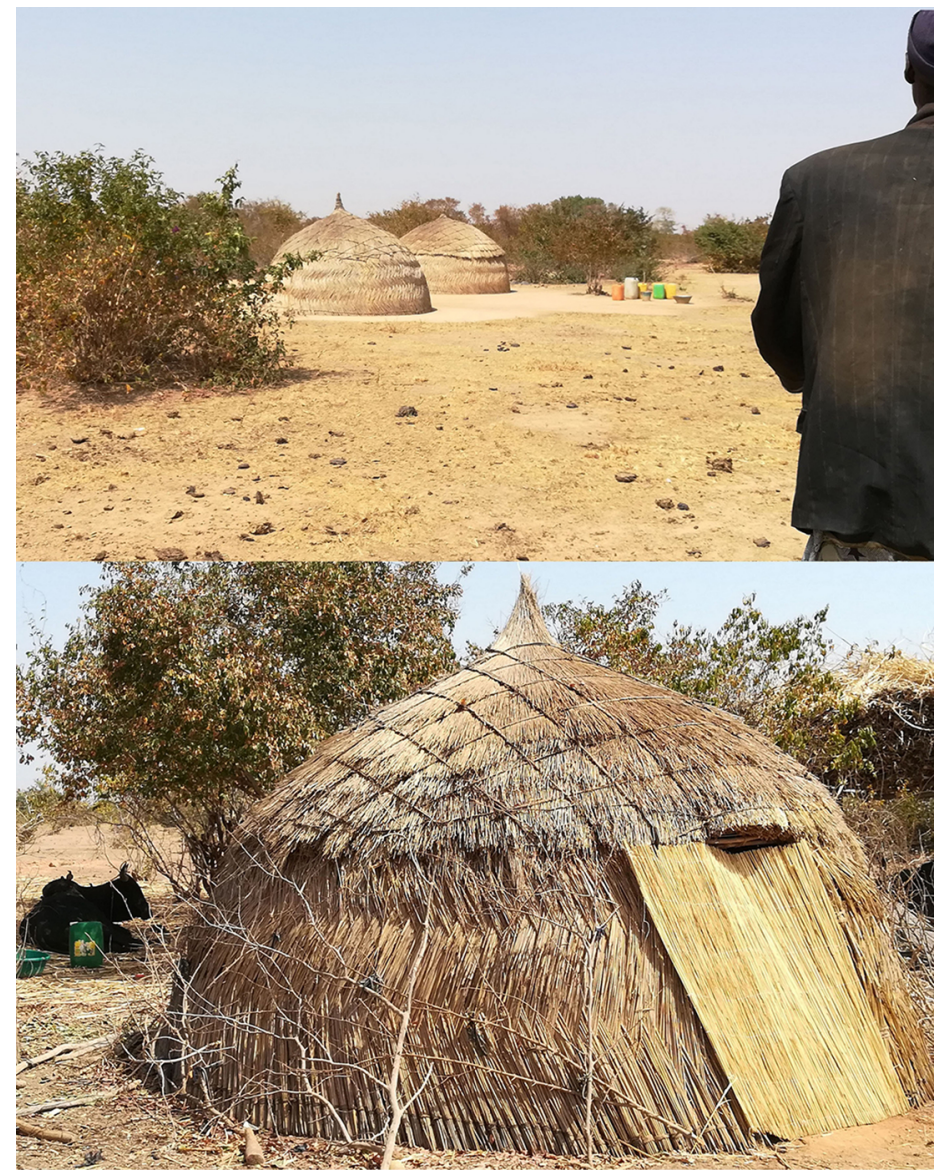

Fotografía de María Aguilar Sánchez, 2017. 


\section{Aspectos formales y materiales utilizados}

La vivienda temporal peul de la zona del Sahel del norte de Burkina Faso ha pasado inadvertida en las investigaciones de Piesik (2017), donde sólo se describen las estructuras nómadas desde Chad hasta Somalia. Sin embargo, la sencillez en la configuración y el modo de construcción de esta particular vivienda le confieren un valor nada desdeñable que hemos considerado conveniente analizar en este estudio. Las viviendas visitadas durante el trabajo de campo tienen la morfología de un iglú, pero están construidas a base de esteras de paja.

La vivienda peul está conformada por varias unidades habitacionales denominadas casserone ${ }^{10}$ que disponen de una planta circular de tres a seis metros de diámetro (imágenes 4 y 5 ). La vivienda visitada, con forma de una semiesfera perfecta, tenía un diámetro de $4 \mathrm{~m}$ y una altura de $2 \mathrm{~m}$. Los casserones se agrupan en torno a un espacio común que generalmente no está cerrado ni delimitado, por lo que se procura que los animales se mantengan alejados de las habitaciones.

La morfología base consiste en una estancia de dimensiones variables, cuyos muros son construidos mediante ramas entrelazadas o esteras que después se cubren con hojas de árboles y paja.

Los peuls de la brousse ${ }^{11}$ hacen sus casas con materiales que se encuentran en el mismo entorno, como los siguientes:

- Ramas de árboles y cordeles para configurar la estructura.

- Esteras de paja y, a veces, tierra, heces de vaca y agua para construir los muros y revestimientos.

Los criterios de selección de plantas utilizadas en la construcción de las viviendas de esta zona y otras del norte de

${ }^{10}$ Se denomina casserones a las construcciones generalmente de planta circular de una sola estancia.

${ }^{11}$ Término que define el campo en Burkina Faso, que en estas zonas suele ser un paisaje de estepa con muy poca vegetación. 
Burkina Faso fueron descritos por Ganaba, Ouadba y Bognounou (2004).

IMAGEN 3. Planos de planta

y sección de vivienda peul, Burkina Faso
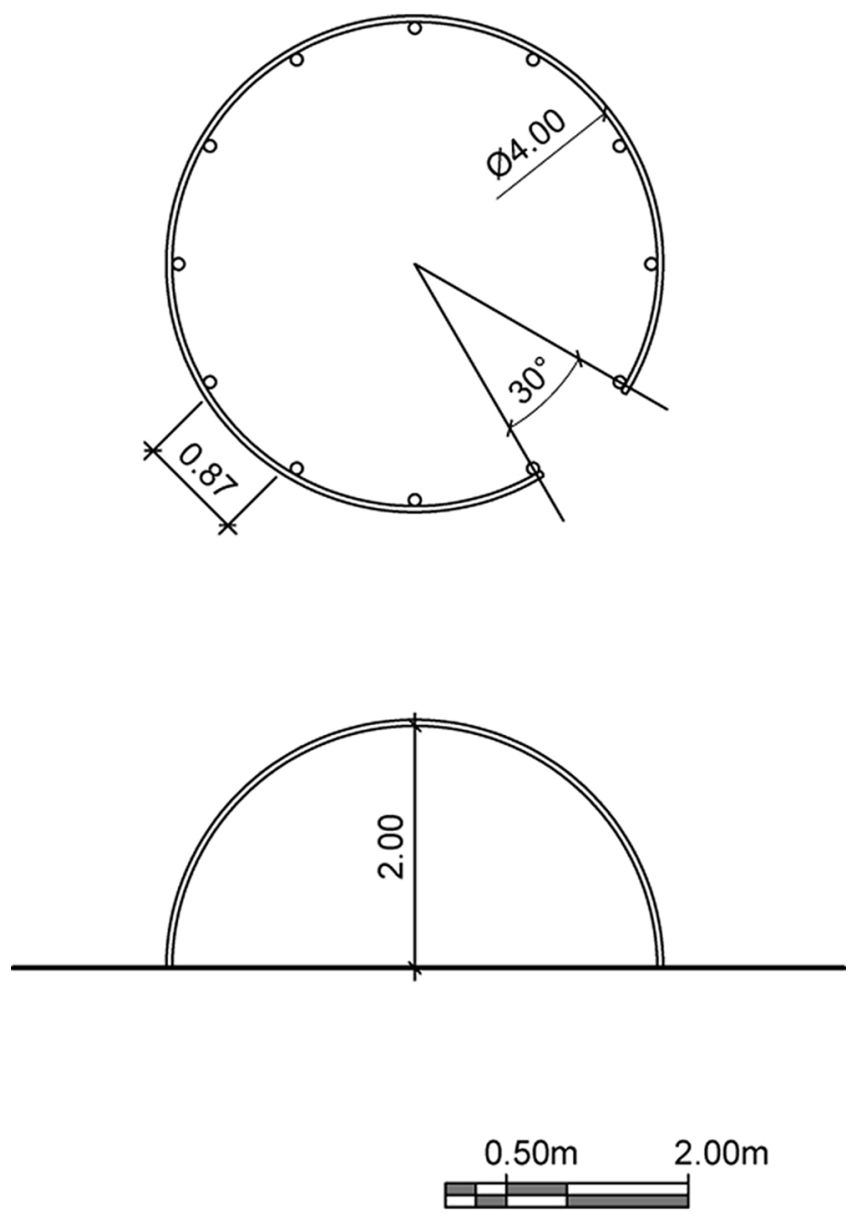

Elaboración propia, 2019. 
Imagen 4. Vivienda peul del Sahel burkinabé, Burkina Faso

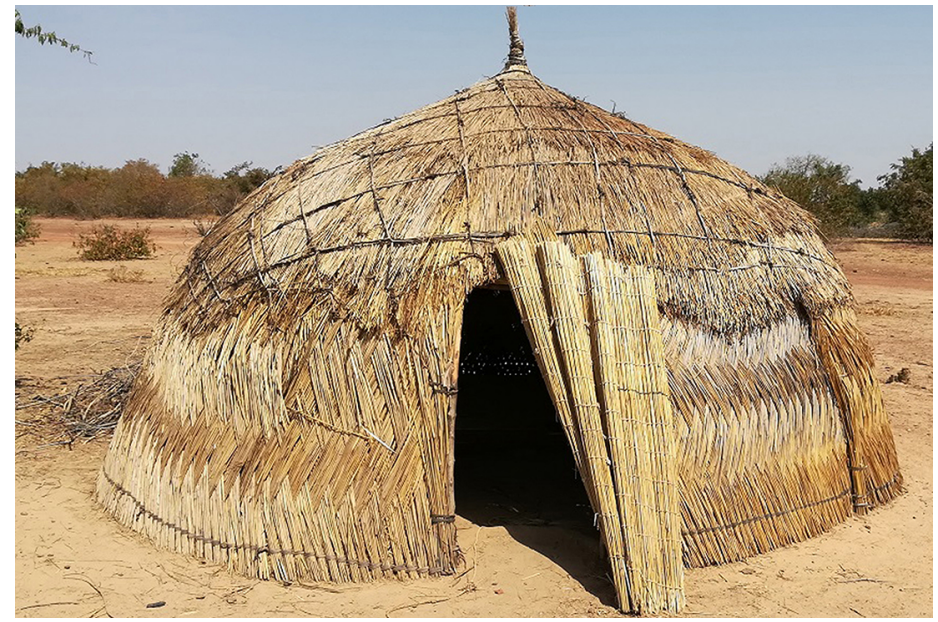

Fotografía de María Aguilar Sánchez, 2017.

Técnicas y sistemas constructivos

Los criterios y las técnicas para construir las viviendas son muy simples, pero se requiere cierta destreza y conocimiento de los elementos y los sistemas constructivos para garantizar una adecuada habitabilidad. Una vivienda puede ser edificada con el trabajo de sólo dos personas expertas en un plazo de cuatro días si se selecciona adecuadamente el lugar y se tiene disponibilidad en el entorno de los materiales requeridos. En la velocidad y la economía de los medios es donde radica la verdadera utilidad y razón de ser de estas viviendas.

Ciertos materiales exigen un proceso antes de su puesta en obra. Por ejemplo, es necesario seleccionar correctamente la madera, dejarla secar, cortarla, recoger el secco, ${ }^{12}$ fabricar las esteras, preparar los cordeles, etcétera (Kéré 1995, 24-26).

${ }^{12}$ El secco es la palabra específica que designa en este caso pequeñas ramas de paja recogidas en el campo para hacer ramilletes. 
El primer paso es marcar la dimensión deseada del módulo circular sobre el lugar seleccionado. Se desbroza el terreno y se delinea un círculo perfecto. Se marca la ubicación de la madera estructural y la entrada principal. A continuación, se levanta la estructura con ramas de longitud, grosor y edad adecuados, que luego se introducen en el suelo unos $30 \mathrm{~cm}$. Dichas ramas estructurales se disponen con una separación constante, igual al ancho de una puerta, para asegurar la estabilidad de la estructura en los $360^{\circ}$ (imagen 3).

Los muros conformados con follaje o paja se elevan sobre soportes de madera que les proporcionan rigidez sin necesidad de ningún cimiento o excavación. Algunos muros de paja son revestidos posteriormente con tierra, aunque lo más habitual es cubrirlos con varias capas de esteras hasta conseguir el grosor adecuado. Finalmente, se refuerzan las zonas de la cubierta hasta obtener un aspecto homogéneo en el que se integran muros y cubierta como un solo conjunto (imagen 5).

En algunos casos, las cubiertas se realizan con hojas, o bien con paja o cañas ${ }^{13}$ sobre ramaje o listones de madera del campo atadas con cordeles de fibras vegetales. Para el suelo de la vivienda se utiliza la misma tierra del lugar, lo cual es una de las razones por las que es tan importante elegir bien la ubicación y preparar la zona antes de la puesta en obra. El interior a veces se decora con secco, nattes, ${ }^{14}$ tejidos o adornos, aunque generalmente el ornamento es superfluo en estas construcciones, cuyo carácter práctico y temporal se hace completamente evidente.

\footnotetext{
${ }^{13}$ Existen varios tipos de paja o caña utilizados en construcción.

${ }^{14}$ Esteras para tumbarse (véase cama de imagen 5). En las ciudades también se utilizan de plástico.
} 
IMAGEN 5. Interior de vivienda peul del Sahel burkinabé, Burkina Faso

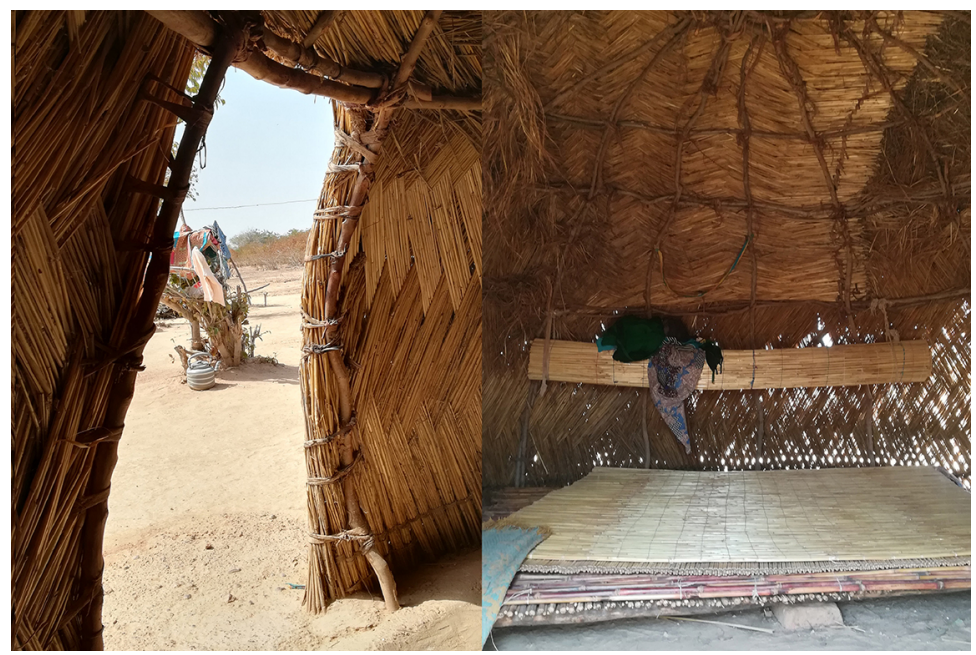

Fotografía de María Aguilar Sánchez, 2017.

\section{La vivienda moaga}

\section{El pueblo mossi}

Los mossis son uno de los grupos étnicos mayoritarios de Burkina Faso y representan casi la mitad de la población, ${ }^{15}$ y el mooré es una de las lenguas más habladas por los burkinabés (Zongo 2004, 20). Aunque conviven en el mismo país, el origen de los mossis es muy diferente al de los peuls, pues proceden de la región sur (actual Ghana) y tienen otro modo de vida no trashumante (Marc 1909).

Varios reinos dieron origen a los mossis en el actual territorio de Burkina Faso. Eran gobiernos independientes que constituían un mismo pueblo y compartían similares costum-

${ }^{15}$ La mayoría de los autores estima 48\% del total del país. 
bres habitacionales y prácticas religiosas. El primero de estos reinos fue el de Tenkodogo, fundado en 1120 por Ouedraogo, el Naaba (Ouattara y Some 2009, 24). ${ }^{16}$ Ouedraogo era hijo de la princesa Yennenga, descendiente de los Moose Gambaga, que durante el siglo XII gobernaban en el norte del actual Ghana (Zongo 2004, 122)

Los principales reinos mossis fueron Tenkodogo, Yatenga y Wogodogo. Desde su migración a estas tierras, los mossis se aliaron con la población autóctona que encontraban, pues su finalidad era mantenerse fuera de la soberanía del Imperio songhai (Izard 1965). La particularidad de la expansión de los mossis reside tanto en la fiereza de su pueblo y su caballería como en las alianzas con los pueblos que iban encontrando. En este sentido, la historia anterior al colonialismo no menciona que sus conquistas se hubiesen realizado con operaciones armadas en contra de las poblaciones indígenas, sino que, más bien, hace referencia a los acuerdos a los que llegaban. Los mossis constituían sus reinos a través de alianzas de personas no sumisas frente a los imperios vecinos (Beucher 2015, 105-124).

\section{Tipología de vivienda y modos de habitar}

Varias etnias de Burkina Faso además de los mossis utilizan la tipología de vivienda de planta circular con muros de adobe y cubierta de sección cónica realizada con paja (imagen 6). La encontramos también en las culturas bissa y gourmantché, establecidas en el centro y este del país, así como entre las culturas senoufo, goin y tella, del sur y la región de Cascades. Kiéthega (2006) especifica que en el hábitat de "planta circular y cubierta cónica de paja" cada etnia refleja pequeñas diferencias de construcción, pero la morfología base y los materiales son los mismos.

${ }^{16}$ Significa "rey" o "jefe” en la lengua mooré del pueblo mossi. 
IMAGEN 6. Vivienda moaga de planta circular y cubierta de paja, Burkina Faso

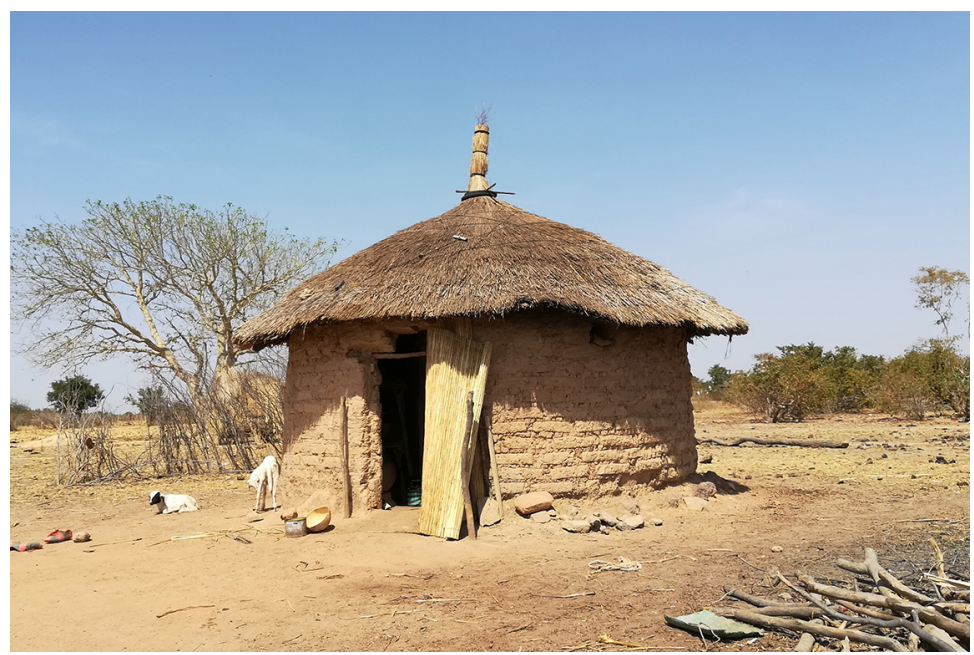

Fotografía de María Aguilar Sánchez, 2017.

Hay variantes de esta tipología en función de cada cultura local. Por ejemplo, los senoufos, los goins y los turkas hacen las cubiertas con varias pendientes debido a la alta pluviosidad de los lugares que habitan. La principal diferencia entre la vivienda gourmantché y la mossi radica en la altura de la cubierta, que suele ser más baja en la primera que en la segunda.

Aunque esta tipología la utilizan diferentes culturas, ${ }^{17}$ es conocida como "vivienda moaga" y suele estar compuesta por varios casserones ${ }^{18}$ dispuestos alrededor de un espacio central de forma más o menos ovalada. El conjunto está delimitado por un muro con una sola puerta. hábitat.

${ }^{17}$ Incluso los peuls burkinabés que se sedentarizan utilizan esta tipología de

${ }^{18}$ Cada adulto suele tener su propio casserone, y cuando la familia es poligámica, las mujeres tienen también cada una el suyo. 
Aspectos formales y materiales utilizados

La vivienda moaga tiene una planta circular de $2.5 \mathrm{~m}$ de diámetro por $3.24 \mathrm{~m}$ de altura y está cubierta por una estructura cónica de paja. La puerta es ojival y tiene $50 \mathrm{~cm}$ de ancho por $1.5 \mathrm{~m}$ de alto. Es el único hueco de la vivienda, ya que no hay ventanas (imagen 7). El resto de las viviendas visitadas tenía dimensiones similares en diámetro, altura de la cubierta y puerta de acceso.

El patriarca o jefe y su mujer tienen cada uno su propia habitación casserone. A veces, si la familia es poligámica, cada mujer tiene también su propia cocina en una pieza cerrada, pero generalmente se cocina al aire libre, en el espacio central del conjunto. Debe tenerse en cuenta que la estación seca dura nueve meses, por lo que es posible cocinar en el exterior casi todos los meses del año.

El espacio común está conformado principalmente por el patio central, alrededor del cual se disponen los diferentes módulos del conjunto. En ese espacio exterior de la casa se ubica, además de la cocina, uno o varios hangares donde se descansa durante las horas de más calor. Debido a las altas temperaturas en este clima, estar a la sombra y aprovechar la mínima brisa es una necesidad. Para la construcción de estos hangares se utiliza una estructura básica de madera, compuesta por algunos pilares y un tejado plano que se cubre con paja o follaje.

Las viviendas disponen de diferentes tipos de equipamiento, dependiendo de si las familias se dedican más a la ganadería o a la agricultura. Aunque la mayoría son agricultoras, suelen tener algunos animales como gallinas, burros y cerdos (según la religión). Los graneros donde guardan las provisiones alimentarias suelen ser parecidos a las viviendas, pero de menor tamaño. En consecuencia, la cubierta cónica no necesita una estructura de madera como soporte. 
IMAGEN 7. Planos de sección, alzado y planta de vivienda moaga visitada, Burkina Faso
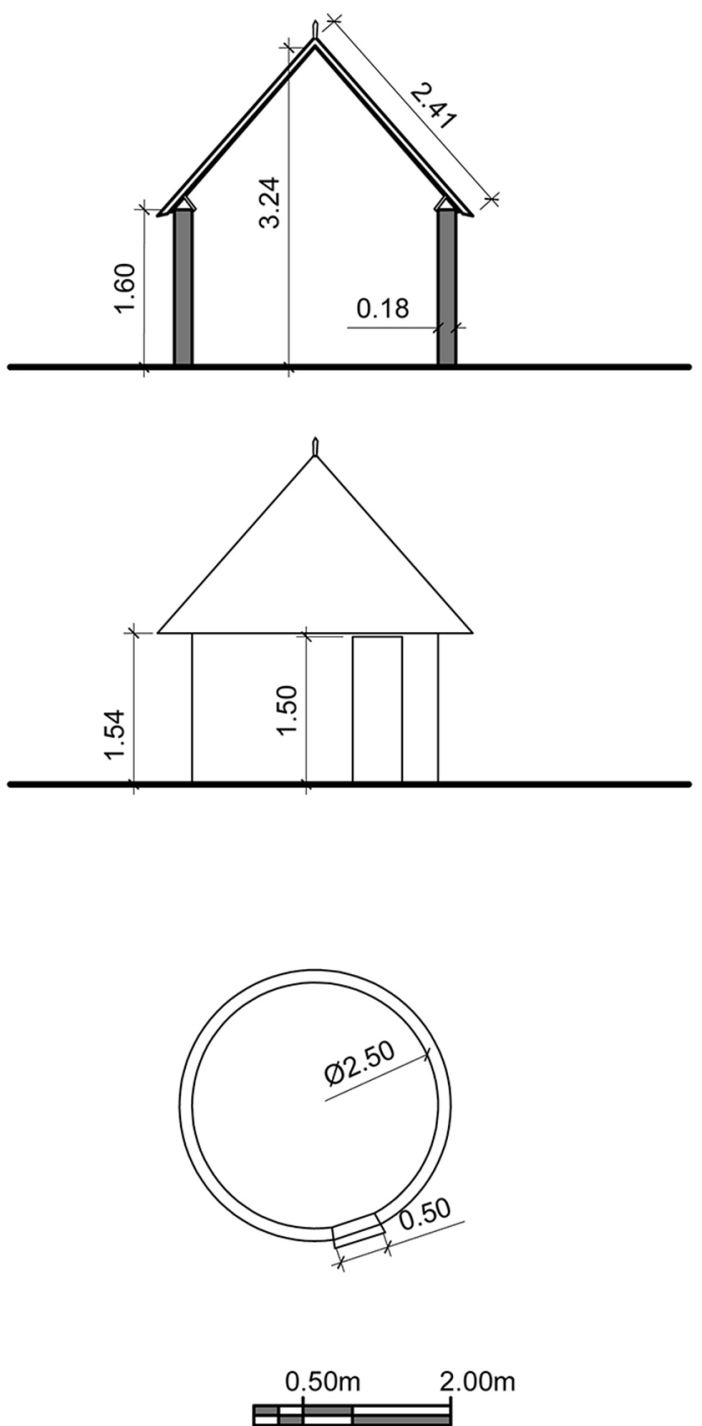

Elaboración propia, 2019. 


\section{Técnicas y sistemas constructivos}

Todos los materiales para la construcción moaga están disponibles en la naturaleza circundante, aunque algunos requieren un proceso de preparación antes de su puesta en obra. Por ejemplo, es necesario fabricar los ladrillos de adobe, secar la madera, tejer las esteras ${ }^{19} \mathrm{y}$ trenzar las cuerdas de $d \hat{a} .^{20}$

Para los muros se utiliza tierra, agua y a veces paja. Las paredes están construidas básicamente con tierra, es decir, bloques de tierra comprimida o adobe (Hema et al. 2017, 536). Para los revestimientos de los muros la materia prima es barro, excremento de vaca, carbón (cenizas) y agua.

Para la cubierta se busca madera en el entorno y algún tipo de aceite o ungüento para ahuyentar las termitas y otros insectos. También se recoge secco, hierbas, cortezas de $d \hat{a}$, plantas trepadoras o fibras vegetales (Zongo 2004). Todos estos materiales también los venden pequeños artesanos que los ofrecen ya elaborados en algunos mercados de los pequeños pueblos (imagen 8).

La construcción de la casa moaga se realiza en diferentes fases. En primer lugar, se selecciona y extrae tierra tipo laterita ${ }^{21}$ para la fabricación de ladrillos de adobe con pequeños moldes cuadrados de madera. Con los ladrillos se hace el cerramiento de la vivienda de forma cilíndrica. Se utiliza adobe para el enfoscado de llagas y tendeles. Los muros se revisten con una mezcla de tierra, agua y carbón que les da un aspecto homogéneo y los protege de las lluvias. También es importante dar un tratamiento previo al interior de los muros (imagen 9). El suelo de la vivienda se compacta mediante pequeños golpes con ramas de secco para obtener una superficie lisa y homogénea.

\footnotetext{
${ }^{19}$ Las esteras se elaboran con diferentes tipos de pajas de gramíneas y se utilizan como las esteras de esparto en la cultura mediterránea.

${ }^{20}$ Planta anual originaria de África, también llamada chanvre de Guinée en francés o cáñamo en español.

${ }^{21}$ Laterita es el nombre específico del tipo de tierra de la zona; es de color rojizo y se utiliza como material de construcción.
} 
IMAGEN 8. Detalle del faldón de paja de vivienda moaga, Burkina Faso

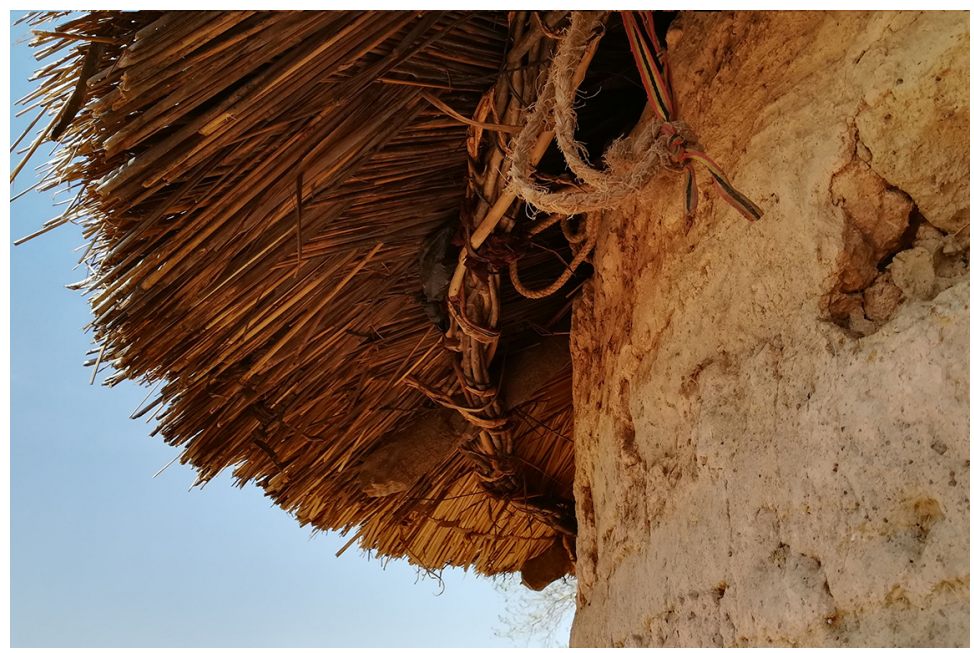

Fotografía de María Aguilar Sánchez, 2017.

En lo que respecta a la cubierta, generalmente se construye en el suelo y posteriormente se coloca sobre los muros. ${ }^{22}$

\section{Factores que condicionan la transformación de los modos de vida mossi y peul}

Si se tiene en cuenta la adaptación al medio de los modos de vida y los saberes y las técnicas de construcción tradicionales de los peuls y los mossis, es evidente que los cambios que se están produciendo en este siglo están afectando decisivamente la vida de estas personas. En este apartado se analizan los

${ }^{22}$ Hay otra técnica antigua llamada colombinage; consiste en amontonar bolas de tierra húmeda en espiral hasta la altura deseada. La casa se construye con forma de vasija, pero este tipo no suele encontrarse en estas zonas de Burkina Faso. 
IMAGEN 9. Interior de vivienda moaga de planta circular y cubierta de paja, Burkina Faso.

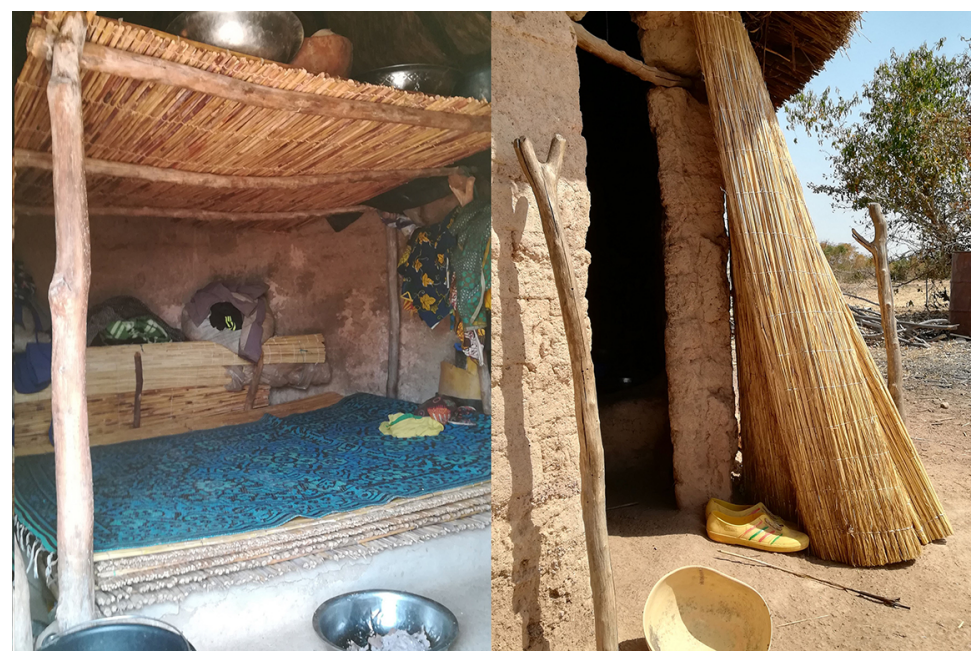

Fotografía de María Aguilar Sánchez, 2017.

principales efectos de esos cambios en el entorno natural y el contexto político-económico que está influyendo en este proceso de transformación.

\section{Escasez de recursos naturales}

Los materiales empleados en la arquitectura vernácula han sido tradicionalmente gratuitos y fáciles de encontrar en el entorno natural. Sin embargo, en la actualidad tienen un costo que dificulta su adquisición. Los precios están aumentando gradualmente, a la vez que disminuye su calidad, y, por tanto, su rendimiento y su durabilidad. Además, la madera y la paja, por su persistencia limitada, deben ser remplazadas periódicamente. A la mala calidad se añade la cada vez menos garantizada 
disponibilidad para reponer la paja. De hecho, los conflictos y la degradación ambiental que señalan Shmite y Nin (2015) se están acentuando debido al auge demográfico y la fuerte disminución de la demanda de la cubierta tradicional.

La escasez de un material básico de construcción como la paja afecta tanto a la vivienda tradicional nómada como a la sedentaria, es decir, tanto a los peuls como a los mossis, y obliga a las poblaciones rurales a buscar soluciones "modernas": techos de vigas cuadradas de madera sobre los que se amarran láminas de chapa corrugada.

Una mayor inversión en el estudio detallado del suelo y la vegetación de esta región permitiría obtener mejores resultados para predecir los problemas de sequía (Breil, Panitz y Schädler 2017, 379-389). Por ello, el desarrollo de sistemas como los de la West African Monsoon (WAM) 23 podría apoyar la disminución de la crisis humanitaria del Sahel, ya que las variaciones en el ciclo estacional de precipitaciones tienen un impacto directo en el hábitat, así como en la disponibilidad de los recursos naturales necesarios para la construcción de las viviendas tradicionales.

\section{Nuevos materiales}

Los problemas reseñados inciden en la dificultad para utilizar materiales tradicionales y están llevando a una paulatina sustitución de los sistemas constructivos ancestrales por otros más modernos. La introducción de nuevos materiales tiene repercusiones importantes en la transformación de la morfología de las viviendas. Por ejemplo, el tejado de paja está siendo sustituido por chapas corrugadas, más duraderas, $\mathrm{y}$, por consiguiente, la planta circular tradicional se está sustituyendo por una geometría rectangular (Wyss 2005, 14). Aunque las chapas tienen un

${ }^{23}$ En español, Monzón del África Occidental, sistema de estudio climático que determina las precipitaciones en el Sahel. 
mayor costo inicial, la mayoría de las familias prefiere esta opción para no tener que rehacer la cubierta a los pocos años. Sin embargo, la chapa corrugada es un material importado que genera graves problemas térmicos. Debe considerarse que los valores de radiación solar sobre las cubiertas son muy elevados en Burkina Faso debido a su baja latitud (en torno a $13^{\circ} \mathrm{N}$ ). Al consiguiente problema de falta de confort en las viviendas hay que añadir el gran déficit de aislamiento acústico de este tipo de chapas.

El simple cambio de material de la cubierta está produciendo una nueva tipología de vivienda, ya que la rigidez de la lámina corrugada y su geometría rectangular son incompatibles con la forma circular y ovalada tradicional, ${ }^{24}$ por lo que las viviendas circulares van desapareciendo a favor de las rectangulares. Las transformaciones morfológicas se producen a diferentes velocidades, por lo que a menudo se encuentran conjuntos mixtos, es decir, casserones tradicionales y módulos rectangulares cubiertos con chapa en el mismo conjunto habitacional.

La vivienda vernácula moaga se adapta al clima y tiene importantes valores patrimoniales en los que se refleja una tradición milenaria de una cultura única. Sin embargo, un conjunto mixto con materiales no adaptados a las condiciones locales genera pérdida de identidad cultural y graves problemas de habitabilidad y confort ambiental. Asimismo, tienden a convertirse en slums, ${ }^{25}$ ya que muchos se construyen y consolidan en la periferia de las ciudades de manera precaria y carecen de ciertas condiciones básicas, como agua potable y saneamiento. La catalogación de viviendas como slums realizada por Sugranyes (2011), y sus connotaciones negativas y sus problemas derivados, se ven reflejados en los nuevos asentamientos resi-

${ }^{24}$ Hay cubiertas cónicas de chapa corrugada en otros países de África, pero las que se comercializan en Burkina Faso a precio asequible para la población tienen poca flexibilidad.

${ }^{25}$ Área de una ciudad con infraviviendas o asentamientos inestables donde las casas se encuentran en mal estado. 
denciales que se han conformado en ciudades como Uagadugú: son precarios y tienen pocas probabilidades de permanecer a largo plazo, ya que los gobiernos correspondientes tienden a tomar medidas para erradicarlos con el pretexto de legalizar los terrenos donde se asientan. Se trata de una cuestión muy debatida en la actualidad, ya que afecta a numerosos países con altos índices de pobreza.

\section{Transformación del régimen de propiedad del suelo}

Como ya se señaló, la incorporación de nuevos materiales en tipologías tradicionales ha dado pie a viviendas mixtas que pueden ser catalogadas como slums, y, desafortunadamente, la legalización de los terrenos donde se desarrollan forma parte de una estrategia mundial respaldada por ONU-Hábitat, entre otras organizaciones (Sugranyes 2011, 27-49). Esta intrusión de otros países en cuanto a la gestión del suelo no es una novedad: "En el trazo de fronteras que resultó de los procesos de colonización y descolonización, el desierto quedó dividido y administrado por capitales lejanas" (Mendoza 2013, 19). De hecho, la estructura del mapa político de la mayoría de los países que han sido colonizados en África no corresponde al mapa de etnias y de lenguas (Zongo 2004, 20), lo que ha provocado la división de pueblos, que ahora pertenecen a naciones distintas.

Del análisis de las tipologías de viviendas seleccionadas, hemos deducido que el modo de vida seminómada tradicional de los peuls está estrechamente relacionado con el régimen de propiedad del suelo (Wyss 2005, 16). En este sentido, conviene aclarar que el concepto de propiedad, tal y como lo conocemos en Occidente, no existía antes de la colonización. De esta forma, las culturas nómadas del Sahel podían establecer acuerdos y concesiones con los grupos sedentarios con los que entraban en contacto (Ouattara y Some 2009). Actualmente, estos pueblos encuentran menos áreas donde asentarse de manera temporal, y son conscientes de que cada vez será más complicado 
mantener su modo de vida tradicional, por lo que tienden a sedentarizarse. Las nuevas generaciones peuls están, por lo tanto, claramente condicionadas por el nuevo modelo urbanístico neoliberal, y los asentamientos informales encuentran grandes dificultades para consolidarse y permanecer en el futuro, sobre todo los que se ubican en la periferia de las principales ciudades, como Uagadugú o Bobo-Dioulasso. ¿Qué podemos esperar de una cultura cuyo modo de vida no contempla la propiedad de la tierra? Evidentemente, teniendo en cuenta la tendencia mundial a la legalización de la propiedad, sólo veremos la paulatina desaparición de esta práctica de seminomadismo.

Como señala Mac Donald (2011), la población más desfavorecida conoce las ventajas de los bienes y los servicios urbanos que proporciona la proximidad a las ciudades. Sin embargo, los pueblos mossi y peul son depositarios de una experiencia secular de adaptación al entorno natural que ha generado una identidad cultural propia y que los distingue de los asentamientos urbanos analizados por este autor. Las soluciones arquitectónicas que han generado son un reflejo de sus valores culturales y les proporcionan identidad y cohesión (Dialla 2005), a tal punto que la permanencia de estas soluciones es clave para su supervivencia como pueblo. Estas viviendas vernáculas dependen directamente de los materiales que se encuentran en el campo sin necesidad de someterlos a grandes procesos de transformación industrial, por ello el modelo de desarrollo mundial no es compatible con estos modos de habitar (Rist 2002, 97-110). En este sentido, las familias entrevistadas durante esta investigación son perfectamente conscientes de que están siendo forzadas a adaptarse al modelo de propiedad privada de la tierra, así como al sistema económico mundial.

\section{Conclusiones}

Las tipologías de viviendas tradicionales de los peuls y los mossis constituyen un patrimonio secular que los identifica como 
pueblo, pero que se está perdiendo por el impacto del entorno y el contexto actual. Los modos de habitar se transforman de modo diferente en cada cultura, aunque con algunas causas y efectos comunes. Mientras los pueblos peuls se vuelven cada vez más sedentarios por la escasez de tierras libres donde instalarse, los mossi modifican su vivienda tradicional con la introducción de nuevos materiales, como la chapa corrugada. En las construcciones en ambos casos se resiente la falta de recursos naturales como la madera y la paja, lo que obliga a los grupos a buscar alternativas que modifican su vivienda y sus modos de habitar.

Este análisis ha permitido esclarecer importantes cambios en el entorno y el contexto de Burkina Faso, los cuales han provocado transformaciones en los modos de vida e influido decisivamente en la pérdida del patrimonio arquitectónico.

Destacan las importantes alteraciones que el cambio climático ha traído al entorno natural del Sahel y sus áreas limítrofes, territorios donde viven los peuls y los mossis. Esto se traduce en un aumento de sequías, disminución del periodo de lluvias y cambios drásticos de clima que afectan los cultivos de los mossis y al ganado de los peuls. También hay una escasez de recursos naturales que se manifiesta en la falta de materiales para la puesta en obra de las viviendas y, por lo tanto, de las posibilidades de mantener las técnicas de construcción tradicional.

El nuevo contexto político y económico de globalización afecta gravemente a las economías y los países más pobres del mundo, entre los que se encuentra Burkina Faso. Esto ha implicado la aparición de nuevos materiales de mayor durabilidad y resistencia, pensados para responder a la demanda de soluciones para la población, pero que conllevan un cambio en la morfología y, por tanto, en los comportamientos. Estos materiales deben ser estudiados en cuanto a confort térmico y acústico, y en cuanto a sus repercusiones en la salud de las personas. Este contexto influye en los modos de vida locales a favor del sector empresarial y del mercado internacional, sin tener en cuenta 
las consecuencias en cuanto a la sostenibilidad medioambiental y la integridad de los valores culturales y sociales.

Por último, consideramos que el neocolonialismo se hace cada vez más presente en Burkina Faso y agrava los problemas surgidos con el cambio de régimen del suelo introducido durante la colonización francesa. El actual sistema de propiedad de la tierra implica la asignación de propietarios legales con titularidad en todas las tierras. En el caso de los peuls, cuyo seminomadismo constituye la base de su modo de vida, el cambio a este régimen aumenta las dificultades de acceder a otras áreas y complica los acuerdos para instalarse temporalmente. Por otro lado, este sistema de gestión del territorio busca la eficacia, pero no se ajusta ni al mapa étnico, ni al de lenguas ni, incluso, al de núcleos comerciales en Burkina Faso antes de la colonización francesa.

Todos estos cambios que afectan a los mossis y a los peuls, unidos a otros más generalizados, como el aumento demográfico o los conflictos terroristas de los países del Sahel, auguran el abandono de prácticas tradicionales a favor de aquellas que determine el mercado internacional. Esta situación puede conducir a la pérdida de patrimonio arquitectónico y de valores culturales únicos. Por este motivo, los estudios encaminados a revitalizar e incorporar la arquitectura vernácula a la cultura moderna deberían ser parte del debate actual sobre desarrollo urbano en Burkina Faso. En este sentido, la experiencia secular de adaptación al entorno natural con los recursos disponibles en cada lugar constituye no sólo un valor patrimonial importante de una cultura o un grupo social, sino también un modelo de vida sostenible en armonía con el medio ambiente, del cual se pueden extraer lecciones para el futuro. La población es consciente de que los nuevos modos de vida al servicio de la globalización son inestables y poco sostenibles a largo plazo; sin embargo, no dispone de alternativas reales a estos problemas, pues se trata de verdadera supervivencia. 


\section{Referencias}

Almodóvar, José Manuel. 2006. La ciudad hispanoamericana desde la composición y el medio ambiente. El caso particular de Arequipa. Sevilla: Junta de Andalucía.

Almodóvar, José Manuel, Griselda Pinheiro y Solange Souza. 2011. Valores culturales y ecológicos de la arquitectura tradicional de Salvador, Brasil. Madrid: Agencia Española de Cooperación Internacional.

BARINGO, David. 2013. "La tesis de la producción del espacio en Henri Lefebvre y sus críticos: un enfoque a tomar en consideración". Quid 16, núm. 3 (noviembre 2013-octubre 2014): 119-135.

BARral, Henri. 1967. Les populations d'éleveurs et les problèmes pastoraux dans le Nord-Est de la Haute-Volta. París: Office de la recherche scientifique et technique outre-mer.

Baux, Stephanie. 2013. "Mise en contexte pour l'enquête pildu du Projet ECAF : le Burkina Faso et la ville de Ouagadougou”. http:// docplayer.fr/5642506-Mise-en-contexte-pour-l-enquete-pildudu-projet-ecaf-le-burkina-faso-et-la-ville-de-ouagadougou.html

BEUCHER, Benoît. 2015. “Trajectoires impériales croisées : l'historicité d'un État africain hybride (pays moaaga, actuel Burkina Faso, fin du XIX siècle à nos jours)". Cabiers d'histoire. Revue d'histoire critique, núm. 128: 105-124.

BREIL, Marcus, Hans-Jürgen Panitz y Gerd Schädler. 2017. "Impact of Soil-Vegetation-Atmosphere Interactions on the Spatial Rainfall Distribution in the Central Sahel”. Meteorologische Zeitschrift 26, núm. 4: 379-389. https://doi.org/10.1127/metz/2017/0819

Dialla, Basga Emile. 2005. "Pratiques et savoirs paysans au Burkina Faso: une présentation de quelques études de cas”. Série documents de travail DT-CAPES núm. 2005-2020. Centre d'Analyse des Politiques Economiques et Sociales, Burkina Faso, julio. http:// www.slire.net/download/1559/pratiques-savoirs.pdf

Ganaba, Souleymane, Jean-Marie Ouadba y Ouétian Bognounou. 2004. "Plantes de construction d'habitations en région sahélienne”. Bois $\mathcal{E}$ forets des tropiques 282: 11-17. https://revues.cirad. $\mathrm{fr} /$ index.php/BFT/article/download/20215/19974/0

Halen, Pierre. 2012. Reseña de "Bâ (Amadou Hampâté), Mémoires. Amkoullel, l'enfant peul. Oui, mon commandant! Sur les traces d'Amkoullel l'enfant peul [Préface de Théodore Monod]. 
Arles : ActesSud, coll. Thesaurus, 2012, 850 p. IsBN 978-2-33000624-2". Études littéraires africaines, núm. 33: 148-149. https:// doi.org/10.7202/1018712ar

Hampshire, Kate y Sara Randall. 2000. "Pastoralists, Agropastoralists and Migrants: Interactions between Fertility and Mobility in Northern Burkina Faso”. Population Studies 54, num. 3: 247-261. https://doi.org/10.1080/713779098

Hema, Césaire M., Geoffrey Van Moeseke, Arnaud Evrad, Luc Courard y Admah Messan. 2017. "Vernacular Housing Practices in Burkina Faso: Representative Models of Construction in Ouagadougou and Walls Hygrothermal Efficiency". Energy Procedia 122 (septiembre): 535-540. https://doi.org/10.1016/j. egypro.2017.07.398

INSD (Institut National de la Statistique et de la Démographie [Burkina Faso]). 1994. Analyse des résultats de l'Enquête Démographique 1991. Première partie : Etat de la population, Habitat et Ménage. Uagadugú: INSD. https://www.odsef.fss.ulaval.ca/sites/odsef.fss. ulaval.ca/files/fonds_gp/c-doc_362_odsef.pdf

IZARD, Michel. 1965. Traditions historiques des villages du Yatenga. París: Centre national de la recherche scientifique.

KÉRÉ, Basile. 1995. Architecture et cultures constructives du Burkina Faso. Villefontaine: CRATerre-EAG.

KIÉTHEGA, Jean-Baptiste, dir. 2006. État des lieux des savoirs locaux au Burkina Faso : ethnobotanique et médecine traditionnelle : pratique et systèmes culturaux : ethnozoologie et santé animale : habitats, matériaux locaux et énergie: artisanat, arts du feu et pratiques funéraires. Uagadugú: Centre d'Analyse des Politiques Economiques et Sociales.

KотTEK, Markus, Jürgen Grieser, Christoph Beck, Bruno Rudolf y Franz Rubel. 2006. "World Map of the Köppen-Geiger Climate Classification Updated”. Meteorologische Zeitschrift 15, núm. 3 : 259-263. https://doi.org/10.1127/0941-2948/2006/0130

Lefebvre, Henri. 1974. "La production de l'espace". L'Homme et la société, núm. 31-32: 15-32. https://doi.org/10.3406/homso.1974.1855

Mac Donald, Joan. 2011. "Ciudad, pobreza, tugurio. Aportes de los pobres a la construcción del hábitat popular”. Hábitaty Sociedad, núm. 3: 13-26. https://doi.org/10.12795/habitatysociedad.2011. i3.02 
Marc, Lucien. 1909. Le pays mossi. París: Larose.

MAuss, Marcel. 1905. "Essai sur les variations saisonnières des sociétés eskimos. Étude de morphologie sociale”. Versión electrónica editada por Jean-Marie Tremblay en la colección Les classiques des sciences sociales. Chicoutimi: Université du Québec. https:// doi.org/10.1522/cla.mam.ess4

MendozA, Natalia. 2013. "La crisis del Sahara”. Nexos 34, núm. 424: 19-23. https://www.nexos.com.mx/?p $=15248$

Monnier, Laurent e Yvan Droz, dirs. 2004. Côté jardin, côté cour : anthropologie de la maison africaine. Ginebra: Graduate Institute Publications. https://doi.org/10.4000/books.iheid.2361

Navarro, Vicente Gonzalvo. 2019. "Perspectiva estratégica de la situación en Burkina Faso: el apoyo internacional”. Revista Espanola de Relaciones Internacionales, núm. 10: 98-137.

Ounttara, Ardjouma y Lanko Some. 2009. Analyse des résultats définitifs $d u$ recensement général de la population et de l'babitation de 2006 (RGPH-2006), thème 9 : Croissance urbaine (BFA-INSDRGPH-2006). Uagadugú: Institut national de la statistique et de la démographie. http://www.cns.bf/IMG/pdf/theme_9_urbanisation_fin_f.pdf

PIEsIK, Sandra. 2017. Hábitat: arquitectura vernácula para un planeta cambiante. Traducido por Remedios Diéguez Diéguez. Barcelona: Blume.

RIsT, Gilbert. 2002. El desarrollo: historia de una creencia occidental. Traducido por Adolfo Fernández Marugán. Madrid: Los Libros de la Catarata.

SAGnON, L. Célestin y S. Paul Sawadogo. 2009. Analyse des résultats définitifs $d u$ recensement général de la population et de l'babitation de 2006 (RGPH-2006), thème 10: Ménages et habitations. Uagadugú: Institut national de la statistique et de la démographie. http:// www.cns.bf/IMG/pdf/th_10_menage_et_habitations_fin_f. pdf

SHmite, Stella Maris y María Cristina Nin. 2015. “África al sur del Sahara: conflictos y degradación ambiental en el Sahel”. Cuadernos de Geografía: Revista Colombiana de Geografía 24, núm. 2 (juliodiciembre): 205-219. https://doi.org/10.15446/rcdg.v24n2.50221

Sugranyes, Ana. 2011. "Chabolismo indignante: el hábitat de los que sobran”. Hábitaty Sociedad, núm. 3 (noviembre): 27-49. https:// doi.org/10.12795/habitatysociedad.2011.i3.03 
Wyss, Urs. 2005. La construction en 'matériaux locaux'. État d'un secteur à potentiel multiple. Uagadugú: Direction du développement et de la coopération. https://jaga.afrique-gouvernance.net/_ docs/resource_fr_65911.pdf

Zongo, Bernard. 2004 . Parlons mooré : langue et culture des mossis, Burkina Faso. París: L'Harmattan.

María Aguilar Sánchez es doctoranda en arquitectura por la Universidad de Sevilla y en sociología por la Université Ouaga 1 Professeur Ki-Zerbo (Burkina Faso). Fue miembro de órganos representativos de la universidad ante el Ministerio de Educación de España y participó también en el Aula de Investigación de Arquitectura Sostenible y el grupo Hábitat y Desarrollo. Asimismo, es miembro del grupo de investigación RNM-162: Composición, Arquitectura y Medio Ambiente, de la Universidad de Sevilla. Ha desarrollado estancias en la Facultad de Tecnologías de la Universidad de Tolima (Ibagué, Colombia) y en la Facultad de Ciencias Sociales de la Université Ouaga 1, y ha colaborado con el laboratorio de Recherches Pluridisciplinaires en Sciences Humaines. Se especializa en cooperación y redes internacionales, y ha ejercido como arquitecta en África subsahariana y como coordinadora de proyectos de cooperación al desarrollo en Burkina Faso y Senegal.

https://orcid.org/0000-0002-3843-0220

maragusan@alum.us.es

José Manuel Almodóvar Melendo es profesor titular en la Escuela Técnica Superior de Arquitectura y Grado en Estudios de Asia Oriental de la Universidad de Sevilla. Tiene tres sexenios de investigación reconocidos por la Comisión Nacional de Evaluación de la Actividad Investigadora. Ha sido profesor invitado en Columbia University, University of California, Berkeley; Tsinghua University y Nanjing University (China), 
y Kobe Design University y University of Shiga Prefecture (Japón). Ha dirigido o participado en proyectos de investigación financiados por la Unión Europea y en colaboración con universidades iberoamericanas (México, Perú, Brasil). Es autor de más de 40 publicaciones indexadas. Tiene título en chino, japonés y portugués por el Instituto de Idiomas de la Universidad de Sevilla.

https://orcid.org/0000-0002-4736-750X jmalmodovar@us.es 
